\title{
Accountable Care in the Safety Net: A Case Study of the Cambridge Health Alliance
}

The mission of The Commonwealth Fund is to promote a high performance health care system. The Fund carries out this mandate by supporting independent research on health care issues and making grants to improve health care practice and policy. Support for this research was provided by The Commonwealth Fund. The views presented here are those of the authors and not necessarily those of The Commonwealth Fund or its directors. officers, or staff.

For more information about this brief, please contact:

Karen Hacker, M.D., M.P.H.

Director

Allegheny County Health Department KHacker@achd.net

To learn more about new publications when they become available, visit the Fund's website and register to receive email alerts.

Commonwealth Fund pub. 1756 Vol. 13

\author{
Karen Hacker, Robert Mechanic, and Palmira Santos
}

\begin{abstract}
Following passage of health care reform in Massachusetts, the Cambridge Health Alliance (CHA), a public safety-net health system, began to establish an accountable care organization in an effort to continue its mission and remain financially solvent. In examining how CHA undertook its delivery system transformation, this case study explores the organization's four major strategies: establishing patient-centered medical homes, entering alternative payment arrangements with managed care organizations, launching complex care management, and establishing a partnership with a tertiary care institution. Workforce education and culture change were also core principles. Within two years, CHA had already received National Committee for Quality Assurance patient-centered medical home recognition for six of its primary care sites, and quality metrics demonstrate improvements in these sites compared with others. Moreover, utilization in one managed care organization is trending downward. Challenges persist, however, due in part to fiscal pressures created by state health care reform.
\end{abstract}

\section{OVERVIEW}

The Cambridge Health Alliance (CHA) is a public safety-net health system based in Cambridge, Mass. In 2006, after the state passed its health care reform law, CHA formed an accountable care organization (ACO) to continue its mission and remain financially solvent. An ACO is an integrated health care system made up of hospitals, physicians, and other providers who share the responsibility of caring for a population of patients. The goal is to provide high-quality, coordinated care and to reduce spending by eliminating unnecessary services, with providers potentially rewarded for meeting quality and cost benchmarks. Early ACO pilots have focused on commercial and Medicare beneficiaries; few have addressed the feasibility of implementing ACOs for Medicaid beneficiaries and other vulnerable populations. Safety-net providers, from public hospitals and health systems to community health centers, must transform the way they serve their patients and communities - and do so with fewer resources and more complex patients than traditional providers. ${ }^{1}$

This case study—based on interviews with staff, document review, and financial analysis - examines the market, policy, and organizational factors that led CHA to undertake ACO transformation; highlights key strategies, areas of 
success, and unique challenges; and identifies operational and policy-related factors that could affect the success of transformation for safety-net systems. (See "About This Study," page 15, for complete methodology.)

\section{BACIKGROUND, POLICY CLIMATE, AND FINANCIAL CHALLENGES}

Cambridge Health Alliance is a public, safety-net health care system affiliated with Harvard and Tufts Medical Schools. Its mission is to improve the health of the communities it serves.

CHA serves a combined population of over 380,000 residents in its primary service area (Exhibit 1). It has three hospital campuses, accounting for over 12,000 annual discharges from three emergency departments (EDs), including one free-standing ED; a psychiatric emergency service; two acute inpatient hospital campuses; and five inpatient psychiatric units serving children, adolescents, adults, and the elderly. Exhibit 2 shows the unique number of patients in the primary care centers and the volume or staffed beds in the emergency departments and inpatient settings. Of note, CHA provides 11 percent of all statewide inpatient mental health admissions for Medicaid and uninsured patients.

Cambridge Health Alliance operates 15 licensed ambulatory care sites that range from fullservice community health centers to single specialty practices and school-based health centers. CHA employs over 300 physicians. It has a systemwide ambulatory and inpatient electronic medical record (EMR) platform that was launched in 2005 and completed in 2012. CHA met the Centers for Medicaid and Medicare Level 1 requirements for meaningful use of electronic health records in 2012.

CHA's 99,000 primary care patients are racially and ethnically diverse; more than 40 percent speak a language other than English. They are largely low-income; 60 percent have publicly funded insurance and about 15 percent are uninsured. More than 20 percent of the population has substance abuse or mental health disorders and many more face challenging social circumstances.

\section{Exhibit 1. Cambridge Health Alliance Service Area, 2012}

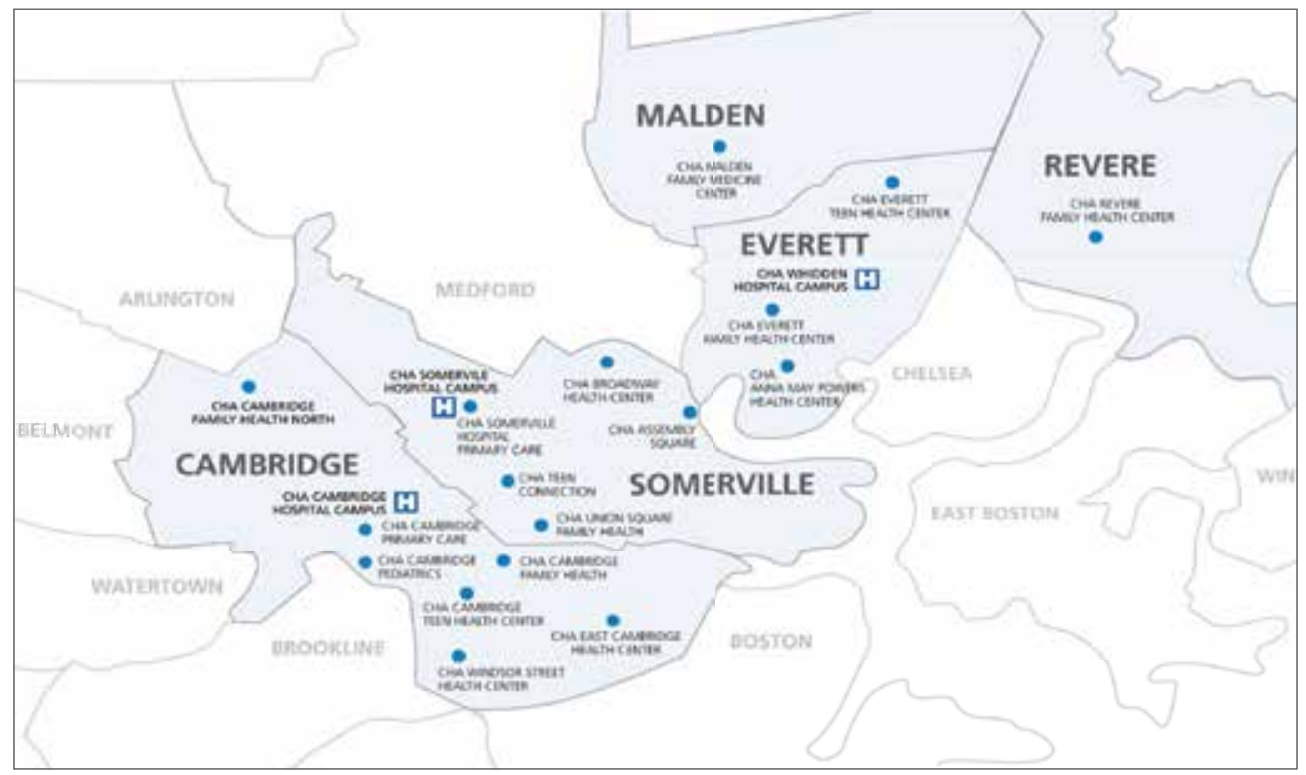

Source: Cambridge Health Alliance Delivery System Transformation Initiative Proposal, updated June 2012 
Exhibit 2. Cambridge Health Alliance Centers and Hospital Campuses

\begin{tabular}{|c|c|c|c|}
\hline Primary Care Centers (2012) & $\begin{array}{l}\text { Unique } \\
\text { Patients } \\
\end{array}$ & Hospital Campuses (2012) & $\begin{array}{c}\text { Volume/ } \\
\text { Staffed Beds }\end{array}$ \\
\hline Cambridge Family Health/North & 9,649 & Emergency Departments & Volume \\
\hline East Cambridge Primary Care & 8,104 & Somerville Hospital & 20,302 \\
\hline Malden Center Family Medicine & 9,495 & Cambridge Hospital & 31,572 \\
\hline Primary Care Unit (TCH) & 7,197 & Whidden Hospital & 44,157 \\
\hline Cambridge Pediatrics & 6,672 & Psychiatric Department & 2,187 \\
\hline \multirow{2}{*}{ Somerville Adult Medicine (Broadway) } & \multirow{2}{*}{8,694} & $\begin{array}{r}\text { Total } \\
\end{array}$ & 98,218 \\
\hline & & Cambridge Hospital Inpatient & Beds \\
\hline \multirow{2}{*}{ Somerville Adult and Family Health } & \multirow{2}{*}{7,311} & Medical/surgical & 50 \\
\hline & & Psychiatric & 45 \\
\hline \multirow{2}{*}{$\begin{array}{r}\text { Somerville Pediatric Medicine } \\
\text { (Broadway) }\end{array}$} & \multirow{2}{*}{7,264} & ICU & 6 \\
\hline & & Maternity/nursery & 28 \\
\hline Union Square Family Health & 7,687 & Total & 129 \\
\hline WH Revere Internal Medicine & 9,302 & Whidden Hospital Inpatient & Beds \\
\hline Windsor Street Health Center & 13,172 & Medical/surgical & 54 \\
\hline Zinberg Clinic & 356 & Psychiatric & 44 \\
\hline \multirow[b]{2}{*}{ Total } & \multirow[b]{2}{*}{95,020} & ICU & 6 \\
\hline & & Total & 104 \\
\hline
\end{tabular}

Source: CHA Patient Database and Delivery System Transformation Initiative (DSTI).

\section{Massachusetts' Health Care Market and Policy Environment}

Health care reform in Massachusetts was the catalyst for CHA's decision to build an ACO. The 2006 health reform law increased access to care through near-universal health insurance coverage. However, nearly 15 percent of CHA's patients-including undocumented immigrants, homeless individuals, and those who had not enrolled in insurance plans-remained uninsured. Additionally, the new Health Safety Net (HSN) fund that replaced the state's uncompensated care pool adopted a Medicare-like payment formula that substantially reduced reimbursements to safety-net hospitals.

In 2008 and 2010, Massachusetts passed laws that laid a foundation for cost control. During that period, a special commission on health care payment recommended the state transition from a fee-for-service model to global payments. ${ }^{2,3}$ Whereas fee-for-service pays for each patient service rendered, global payment pays one amount for all care provided to a population of patients regardless of the amount of service used. Commercial insurers were already adopting this strategy. ${ }^{4}$

In the summer of 2012, Massachusetts passed Chapter 224, a health care control bill, marking the next phase of reform. ${ }^{5}$ The new legislation established a statewide target for total health spending equal to the projected growth of the state economy and promoted the development of patient-centered medical homes (PCMHs) and ACOs through new payment reforms in Medicaid. ${ }^{6}$

With each major policy change, CHA recognized the imperative to move away from fee-forservice and toward global payment and to enhance population health management through an integrated delivery system or ACO. However, to successfully make the transition, CHA needed additional resources. In 2012, CHA received some relief when Massachusetts negotiated its Medicaid 1115 demonstration waiver that includes a new delivery system transformation initiative (DSTI). This program provides incentive payments for projects that support safety-net providers' transition from feefor-service to alternative payment arrangements. ${ }^{7}$ 


\section{Preparing for Transformation}

Cambridge Health Alliance began its transformation in 2009 by developing a vision for where it wanted to be in 2015. Given the forthcoming policy changes at the state and national level, CHA decided to restructure both its delivery system and financing models to successfully continue its mission and remain financially solvent. Health care reform presumed that safety-net providers would shrink as coverage grew, and thus there was ongoing pressure from federal and state government to decrease and ultimately phase out government subsidies.

The framework for transformation is based on the Institute for Healthcare Improvement's "triple aim" 8 of improving the health of a population and patients' experiences of care while lowering costs. CHA began with the aim of transforming ambulatory sites into PCMHs, which would serve as the foundation for the ultimate goal of transition to an ACO model (Exhibit 3).

Soon after it adopted the 2015 vision, CHA created two new leadership positions: vice president for PCMH development and chief administrative officer for ACO development. A temporary PCMH task force with five work groups was established to develop recommendations on education, compensation, performance metrics, and care redesign with broad participation from leadership and frontline staff. In addition, CHA created a leadership academy in 2010 to engage and train staff in ACO and PCMH concepts. In 2011, CHA launched an organizational structure for transformation that established 15 work groups, each focused on addressing key areas of ACO-PCMH clinical and infrastructure development. CHA also joined the Safety-Net Medical Home Initiative, which is sponsored by the Executive Office of Health and Human Services and the Massachusetts League of Community Health Centers.

\section{Exhibit 3. Cambridge Health Alliance's ACO-PCMH Transformation Model}

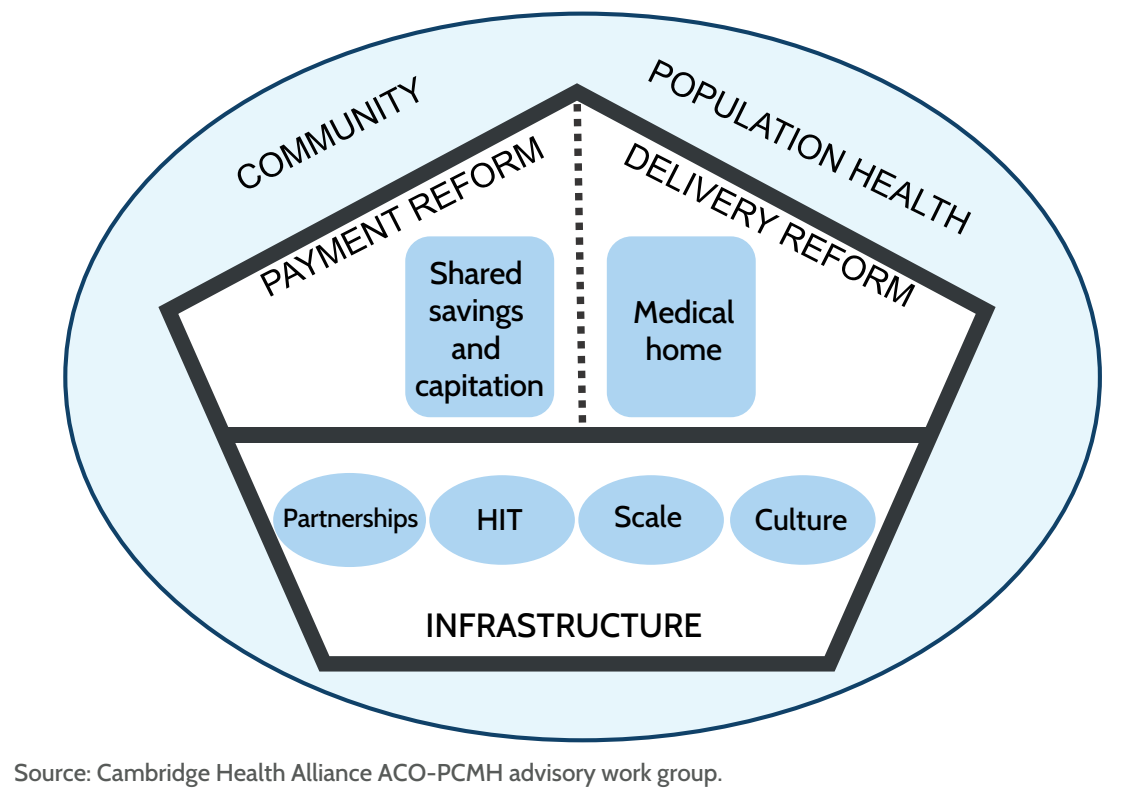




\section{Financial Structure}

Like other safety-net health care systems, CHA's financial structure is complex, and the organization faces financial pressures. As such, a key goal of CHA's transformation efforts is to help stabilize its long-term finances. The ACO-PCMH initiative, however, will not resolve its immediate financial problems. In fact, addressing the current financial issues will likely affect the level of resources CHA can devote to transformation activities.

CHA's delivery system generated operating losses in nine of the past 11 years, and the magnitude of these losses has increased since in 2007 (Exhibit 4). Before 2012, CHA had been able to offset delivery system losses with earnings generated by its wholly owned insurance plan, Network Health. Between 2008 and 2012, the CHA delivery network lost about $\$ 30$ million on average annually, but overall CHA generated average annual surpluses of about $\$ 15$ million. However, the state modified capital requirements that would affect the ownership of Network Health, compelling CHA to sell the plan at the end of 2011. While proceeds from the sale provide CHA with some flexibility, it must now rapidly narrow operating losses to remain financially sustainable.

Cambridge Health Alliance relies heavily on state and federal financial support. In 2012, 32 percent of patient revenue came from supplemental payments (i.e., additional Medicaid payments that subsidize public hospitals) and the Health Safety Net (HSN). The HSN is a state fund set up to pay for essential health care services provided to uninsured and underinsured Massachusetts residents in acute care hospitals and community health centers (Exhibit 5). Overall, about 60 percent of CHA patient revenue comes from Medicaid and other low-income coverage programs, while 22 percent comes from Medicare and 20 percent from private insurers.

Despite massive restructuring to address operating losses, CHA faces important challenges to achieving long-term financial sustainability. These include payer mix, because Medicaid is the predominant payer and traditionally pays less than commercial insurance, and service mix, because CHA does not provide lucrative specialized care, and most of its hospital admissions are for routine medical treatment and psychiatric care, which are poorly reimbursed. Recent state reports indicate that commercial insurers pay CHA in aggregate at about 75 percent of statewide average hospital payment rates. Additionally, CHA has high fixed cost because it maintains three small hospital campuses with full-service emergency departments.

Exhibit 4. Cambridge Health Alliance Total Operating Surplus/(Deficit) (in millions)

\begin{tabular}{lll}
\hline Year & CHA Hospital and Physicians & CHA Consolidated \\
\hline 2002 & $(\$ 13.5)$ & $\$ 0.3$ \\
\hline 2003 & $(\$ 29.8)$ & $(\$ 21.4)$ \\
\hline 2004 & $\$ 2.6$ & $\$ 1.4$ \\
\hline 2005 & $(\$ 12.6)$ & $\$ 6.1$ \\
\hline 2006 & $(\$ 13.6)$ & $\$ 14.0$ \\
\hline 2007 & $\$ 1.6$ & $\$ 1.5$ \\
\hline 2008 & $(\$ 29.4)$ & $(\$ 2.4)$ \\
\hline 2009 & $(\$ 37.0)$ & $(\$ 25.3)$ \\
\hline 2010 & $(\$ 20.1)$ & $\$ 2.0$ \\
\hline 2011 & $(\$ 36.9)$ & $\$ 55.4$ \\
\hline 2012 & $(\$ 28.5)$ & $\$ 44.1$ \\
\hline
\end{tabular}

${ }^{*} \mathrm{CHA}$ Consolidated is the delivery system and the managed care organization.

Source: Massachusetts Center for Healthcare Information and Analysis, Hospital Financial Reports, and CHA Annual Financial Statement. 


\section{Exhibit 5. Cambridge Health Alliance Net Patient Service Revenue, 2012}

Total: \$470 million; $79.7 \%$ Government

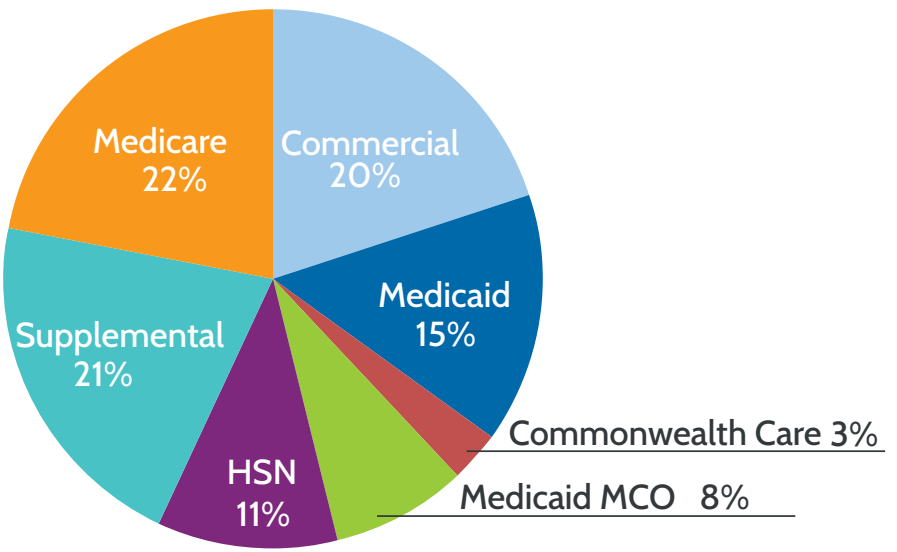

Commonwealth Care is a health insurance program for uninsured Massachusetts residents who meet income eligibility requirements.

Source: CHA Financials.

One important new financial opportunity for CHA is the Delivery System Transformation Initiative (DSTI), part of Massachusetts' most recent Medicaid 1115 waiver. Under the waiver, CHA is eligible for up to $\$ 22.4$ million in annual funding for 2012 to 2014 to support key transformation activities, such as PCMH, behavioral health, and complex care management.

\section{TRANSFORMATION STRATEGY}

\section{Changing the Financial Model}

In response to the policy environment, $\mathrm{CHA}$ accelerated its use of alternative payment models beginning in 2010. First, it entered a shared-risk contract covering about 14,000 Medicaid recipients with its then-owned Medicaid managed care organization (MCO). CHA shares in both savings and deficits relative to agreed-upon annual global budgets. This contract has led CHA to develop infrastructure for managing global payment, such as creating a claims data warehouse for analyzing care patterns and cost. More recently, CHA became a Medicare Shared Savings Program participant in January 2013, which allowed it an additional opportunity to test the global payment strategy with a small population of patients.

By the end of 2013, CHA expects that about half its primary care patients will be covered by programs with alternative payment arrangements like shared risk, shared savings, and pay-forperformance. The largest remaining blocks of CHA patients that will continue to be paid under fee-forservice after 2013 include those who are uninsured but eligible for payment under the Health Safety Net Fund, those in preferred provider organization plans that do not offer global payment programs, and Medicaid recipients not presently eligible for the state's new Comprehensive Primary Care Initiative.

"No matter how efficient we can be, no matter how much savings we can generate, or even how much increased volume we generate-which is a lot of primary care, a lot of mental health, some community hospital stuff, but not the high-end stuff-I think there's still a major concern that the fundamental rate disparities need to be addressed."

- $\mathrm{CHA}$ senior leader 


\section{Building Patient-Centered Medical Home Capability}

Transformation Goal. Cambridge Health Alliance set a goal of transforming each of its primary care sites into patient-centered medical homes (PCMHs) and all obtaining National Committee for Quality Assurance (NCQA) Level III recognition by 2015. This strategy allowed the organization to standardize operations across diverse primary care sites, requiring that all clinics work toward meeting a set of 149 standards in five areas: enhancing access and continuity, identifying and managing patient populations, planning and managing care, providing patients and families with self-care support and community resources, and tracking and coordinating care. Six sites have already achieved Level III recognition. The remaining three sites will apply in early 2015 (Exhibit 6).

PCMH Transformation. CHA used several management strategies to build expertise and facilitate $\mathrm{PCMH}$ transformation. The vice president of PCMH development and a project manager provided support to the sites for developing high-functioning, team-based care. A multidisciplinary workflow committee meets weekly for intensive six-week cycles to analyze existing processes and establish standard workflows. Finally, representatives from all the practice improvement teams meet to spread improvements and develop standardized processes. Staff has benefited from participating in a PCMH learning collaborative, which provided training and resources.

"This [NCQA Level III recognized site] is an incredible example of a medical director partnering with a nursing leader and really transforming the culture together. They had a shared vision and executed an excellent plan. That has to happen in every single one of our sites."

-CHA senior clinical lead

PCMH strategies: empanelment of patients to primary care providers, establishment of care teams and associated processes, coordination of patients with complex care needs, enhanced data reporting for improved performance, and improving patients' experiences. A number of unique issues relevant to safety-net providers surfaced in the process. First, navigating PCMH transformation in a highly unionized environment can require additional negotiation for job changes. CHA managed this by updating the ambulatory registered nurse job description to follow the American Academy

\section{Exhibit 6. Timeline for NCQA PCMH Level III Recognition}

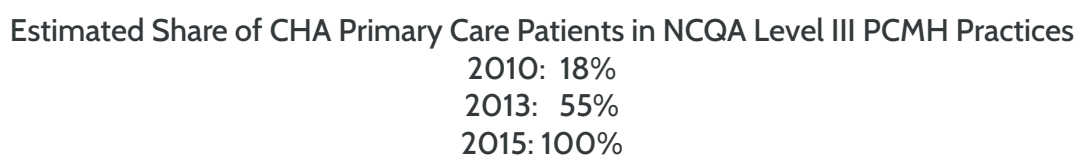

\begin{tabular}{|c|c|c|c|c|c|}
\hline 2010 & 2011 & 2012 & 2013 & 2014 & 2015 \\
\hline \multirow{3}{*}{ Revere Family Health* } & & & Malden Family Health & & $\begin{array}{l}\text { Somerville Family } \\
\text { Health }\end{array}$ \\
\hline & & & $\begin{array}{l}\text { Broadway HC (Adult } \\
\text { \& Pediatrics) }\end{array}$ & & Windsor Street \\
\hline & & & $\begin{array}{l}\text { Everett Family Health } \\
(2013-14)\end{array}$ & & \\
\hline
\end{tabular}

*Originally certified under 2008 NCQA standards and currently applying for recognition under the 2011 standards. Source: Cambridge Health Alliance Administration, Cambridge, MA, 2013. 
of Ambulatory Nursing's competencies and vetting it with the unions. New competencies were also added to other staff roles, including registration clerks and medical assistants. Second, the model of care needed for vulnerable populations requires less focus on chronic disease such as heart disease and diabetes and more on extensive social and behavioral health issues and the social determinants of health experienced by patients. This requires an approach that integrates behavioral health expertise with outreach and community resources.

While CHA had previously attempted to integrate mental health care with primary care in a limited way, PCMH transformation presented a new opportunity to tackle the problem. Two PCMH sites began working to implement an integrated care model for mental health and substance abuse disorders. Both sites have colocated several psychi"I think that the biggest culture change has been having non-provider staff feel so much ownership in the work. That has been a long process of really soliciting ideas about how to do the work, really working side by side instead of top-down."

-Nursing staff member atric and other mental health providers who rotate through the site each week. Based in part on the Improving Mood-Promoting Access to Collaborative Treatment (IMPACT) model of care from Washington State, the approach tailors services depending on levels of mental health issues and acuity. Key strategies include training primary care providers to address mild mental health needs and developing a shared care plan between the behavioral and primary care providers.

\section{Transforming the Workforce and Culture}

Cambridge Health Alliance has adopted multiple strategies to communicate the ACO-PCMH vision throughout the organization. These include regular communication by the chief executive officer and integrating ACO-PCMH activities into regularly scheduled management, clinic, and work group meetings.

Building the case for change was a predominant theme throughout 2011, as was the need to build skills in the workforce. The vice president for PCMH development and a team of administrators worked with site leaders to develop skills in teamwork, motivational interviewing, health literacy, process improvement, and the use of data reports to track outcomes.

Simultaneously, CHA began planning changes in physician compensation for the beginning of 2013 that would reduce the emphasis on productivity and increase financial incentives for patient outcomes and participation in redesign efforts.

CHA conducted a PCMH workforce survey in 2012. The survey found that 90 percent of the workforce understood ACO-PCMH concepts. In addition, staff at NCQA Level III recognized sites were more likely to perceive their practice as operating as a "real team" compared with others (66\% vs. $54 \%){ }^{9}$

CHA has also reviewed and revamped its residency training programs to align with its future as an ACO built on PCMHs. The salient changes in training include a focus on management skills such

"All staff here view patients as their (own) patients, and I know that because that's the language they use. The sophistication and the depth and understanding that medical assistants have in the medical home is real."

-CHA medical director for NCQA Level III recognized clinic 
as systems thinking, complexity management, leadership, collaboration, emotional intelligence, and business.

\section{Implementing Complex Care Management}

CHA established a centralized complex care management (CCM) team in 2011 that initially focused on high-risk patients. Specifically, it pinpointed patients who were expected to be in the top 3 percent in spending of those patients enrolled in a global payment arrangement with a Medicaid MCO. The team has a current census of about 150 patients and manages between 500 and 650 high-risk patients annually. A major focus is connecting patients to primary care and community resources like agencies on aging, supportive housing, and other social services. This connector function should improve as CHA transitions CCM capacity from a centralized program to one located at primary care sites. Regardless of where the CCM is situated, patient "churn" rates remain a major challenge for care coordination. Each year, CHA experiences roughly a 30 percent turnover of its primary care patient population due to patients' losing coverage or moving out of CHA's service area.

Early data suggest the CCM is having a positive impact; an internal analysis of 73 patients found that primary care visits doubled and hospitalizations, emergency department (ED) visits, and total spending declined the first six months they were enrolled in CCM compared with the prior six months.

\section{Creating an Effective Referral Process}

Cambridge Health Alliance is financially responsible for all care delivered to its patients in global payment contracts, whether care is provided by CHA or by external hospitals and physicians. In 2012, CHA established a new referral management process for its 14,000 Medicaid MCO enrollees who receive most of their primary care in CHA but a substantial amount of specialty and inpatient care outside the organization. For example, 65 percent of total inpatient hospital spending and 55 percent of specialist physician spending for these members occurred outside CHA. In comparison, only 14 percent of spending for primary care physicians occurred outside the organization.

Under the new referral guidelines, primary care physicians (PCPs) must first check for appointment availability for CHA specialists, which is available in the EMR. If no CHA specialists are available, the PCP must use a central referral office, which approaches the specialists directly to try to arrange a timely appointment or directs the referral based on a preferred list of external providers.

\section{Establishing Preferred Tertiary and Community Partnerships}

In 2013, CHA announced its affiliation with a tertiary care health system, Beth Israel Deaconess Medical Center. This partnership serves multiple purposes including obtaining better pricing for tertiary services and helping CHA develop new specialty programs, which allows more patients to be served in their communities. It also helps CHA establish a stronger brand, which is imperative in the competitive eastern Massachusetts marketplace.

CHA is also working on expanding partnerships with post-acute care providers. In 2012 it was awarded funding under the Center for Medicare and Medicaid Services' Community-Based Care Transitions Program to work with elder service agencies to coordinate services and reduce readmissions among Medicare beneficiaries. 


\section{Improving Population Health}

CHA developed and implemented a tool that allows the organization to assess the health of the entire population of CHA's 99,000 primary care patients. The tool also allows for patient mapping so CHA can identify the major medical morbidities and compare them to the health indicators in the patients' communities.

Based on this analysis, CHA identified preventive strategies that could yield a return on investment. The first strategy was tobacco cessation; 16 percent of CHA patients are active smokers. CHA began treating tobacco status as a vital sign that is collected at every primary care visit just like blood pressure or weight. Clinics developed a referral mechanism to on-site tobacco-cessation programs. To complement these efforts, $\mathrm{CHA}$ is collaborating on tobacco cessation and smoke-free housing policies with the Cambridge Housing Authority where the majority of residents are CHA patients. This is the first of a range of population-based health strategies that CHA plans to introduce and monitor.

Exhibit 7. Cambridge Health Alliance NCQA Level III Recognized Sites vs. Non-Recognized Sites

\begin{tabular}{|c|c|c|c|}
\hline \multicolumn{2}{|c|}{ Metrics/Indicators } & \multirow{2}{*}{$\begin{array}{c}\text { CHA NCQA III } \\
\text { Recognized }\end{array}$} & \multirow{2}{*}{$\begin{array}{l}\text { CHA Non- } \\
\text { Recognized } \\
65 \%\end{array}$} \\
\hline Cancer & Colorectal cancer screening & & \\
\hline Prevention & Pap screening & $89 \%$ & $82 \%$ \\
\hline \multirow{5}{*}{$\begin{array}{l}\text { Diabetes } \\
\text { Management }\end{array}$} & Blood pressure control & $74 \%$ & $66 \%$ \\
\hline & $2 \mathrm{HgbA} 1 \mathrm{C}$ tests, past 12 months & $88 \%$ & $87 \%$ \\
\hline & HgbA1C $\leq 9$, past 12 months & $84 \%$ & $78 \%$ \\
\hline & LDL $<100$, past 12 months & $64 \%$ & $56 \%$ \\
\hline & $\begin{array}{l}\text { Perfect care: } 1 \text { LDL exam; } 1 \text { eye exam; } 2 \\
\text { HgbA1C tests; } 1 \text { micro-albumin test, all last } \\
12 \text { months }\end{array}$ & $63 \%$ & $59 \%$ \\
\hline \multirow{2}{*}{$\begin{array}{l}\text { Depression } \\
\text { Management }\end{array}$} & $50 \%$ reduction in $\mathrm{PHQ}-9$ score & $29 \%$ & $12 \%$ \\
\hline & $\begin{array}{l}\text { New episodes, antidepressant started, with } \\
3 \text { or more contacts in first } 16 \text { weeks }\end{array}$ & $38 \%$ & $30 \%$ \\
\hline \multirow{3}{*}{ Access } & Rate provider-canceled encounters & $3 \%$ & $4 \%$ \\
\hline & Days to next available appt. after discharge & 6 days & 9 days \\
\hline & & $\begin{array}{l}\text { CHA NCQA III } \\
\text { Recognized }\end{array}$ & MA Multipayer \\
\hline $\begin{array}{l}\text { Patient } \\
\text { Experience }\end{array}$ & Overall Experience with Primary Care Site & $94 \%$ & $88 \%$ \\
\hline
\end{tabular}




\section{EARLY RESULTS: QUALITY, UTILIZATION, AND SPENDING}

\section{Quality}

Early Level III NCQA-recognized sites are outperforming CHA's non-recognized sites on most ambulatory quality metrics, such as cervical cancer screening and diabetes control. In addition, CHA's patient experience scores exceed norms when compared with the primary care practices participating in a statewide multipayer PCMH program (Exhibit 7).

\section{Utilization}

According to at least one Medicaid MCO, there are preliminary findings that show the rate of hospital inpatient admissions at CHA is declining at a greater rate compared with other providers in the MCO network (Exhibit 8). CHA dropped from 134 admissions per 1,000 patient population in the fiscal year 2011 to 127 admissions per 1,000 in fiscal year 2012 and to 113 in the first quarter of fiscal year 2013, compared with a slower rate of decline for the rest of network. While not statistically significant, these preliminary results are promising.

\section{Cost}

CHA conducted an analysis of per-member per-month (PMPM) payments from one Medicaid MCO, focusing on two subpopulations-Medicaid and Commonwealth Care (Massachusetts' expansion population following health reform in 2009). The analysis compared payments between CHA and other network providers for two years following the launch of CHA's global payment model. Results show that the plans' PMPM payments for Medicaid and Commonwealth Care have trended downward. However, given the other changes in the environment - changes in payment rates specific to $\mathrm{CHA}$ and changes in network providers participating in the MCO network-it is difficult to attribute these changes solely to CHA's efforts. For future cost trend analyses, we recommend using complete claims data to disentangle the impact of differences in rate changes and other exogenous factors among network providers.

\section{CHALLENGES}

Cambridge Health Alliance faces important challenges in completing its transformation successfully. Some derive from its role as a safety-net provider while others relate to the inherent challenges of managing organizational change.

\section{Financial Sustainability}

CHA faces an immediate challenge of reversing an annual operating loss of approximately $\$ 28$ million in 2012. Almost 80 percent of CHA's current revenue is from Medicare, Medicaid, and supplemental payments. Over the next decade, Medicare payments are set to rise at historically slow levels under the Affordable Care Act, and Massachusetts is similarly trying to restrain Medicaid spending growth. The strain on government budgets creates uncertainty about the level of supplemental payments that might be available beyond the end of the current Medicaid waiver in 2014.

Due to the immediate pressure for CHA to reduce operating expenses, continued investment in transformation will—at least in the near term—require additional public funding. CHA 
now receives significant funding for its transformation efforts through the Medicaid Delivery System Transformation Initiatives (DSTI) waiver. Now, as CHA attempts to prioritize deficit reduction, resources and management attention may be drawn away from transformation activities.

\section{Defining the Role of the Hospital Within an ACO}

One of CHA's major strengths as an ACO is its network of primary care clinics; however, CHA's finances are dominated by its hospital operation. In 2011, the hospitals accounted for 85 percent $(\$ 370$ million) of CHA's net patient revenue while the physician organization generated about 15 percent of the total (\$70 million). Like many other safety-

"I think at every level right now within $\mathrm{CH}$ A there is this juxtaposition between feeling extremely excited about the groundbreaking work we're involved in and feeling overwhelmed and stressed."

-CHA senior leader net providers, CHA's finances are closely intertwined with its hospital because Medicaid disproportionate share payment formulas are linked to hospital utilization. In addition, CHA's primary care clinics are classified as hospital outpatient departments for Medicaid payment purposes and receive significantly higher fees than they would as freestanding clinics. Therefore, despite severe financial challenges, CHA's affiliation to the hospital is critical to its current revenues.

CHA faces a fundamental dilemma that is common to aspiring ACOs. Should it become an integrated primary care network focused on population health with an aligned hospital, or will it remain a hospital system that includes a primary care network? These approaches require fundamentally different strategies and resource allocation decisions. The former requires viewing the hospital as a cost center while the latter emphasizes generating sufficient volume to maintain the economic viability of the inpatient platform. Leadership is currently grappling with this strategic dilemma.

\section{Exhibit 8. Hospital Admissions at Cambridge Health Alliance Down Compared with Other Providers in One Medicaid Managed Care Organization}

Risk-adjusted admits/1,000 members

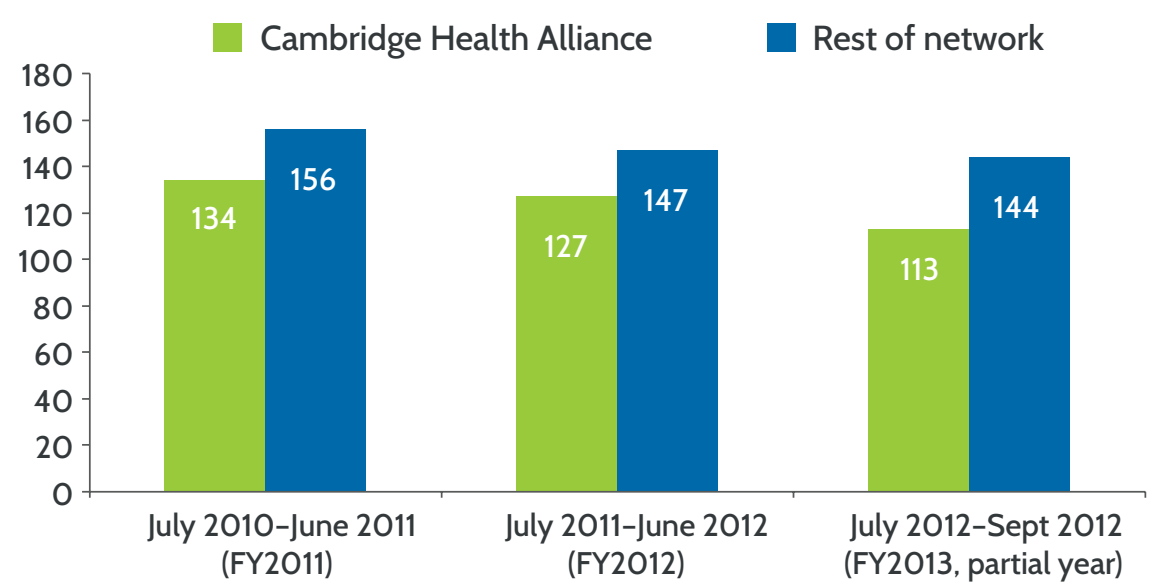




\section{Building a Culture of Action and Accountability}

CHA's culture has historically emphasized consensus, with a broad range of staff and leadership involved in making decisions.

Many of the people interviewed, however,
"Right now, we have a system where everybody contributes their ideas and no one has really clear authority to decide. So it's much easier to get something vetoed than to get it approved."

-CHA physician-leader noted that CHA's culture of consensus and innovation results in slow decision-making, conflicting priorities, and a lack of clarity about who is ultimately responsible for implementing change or achieving specific goals. One indication of this is the wide range of performance metrics used across the organization. While CHA has the advantage of a robust information system, many respondents expressed a feeling of data overload. Different primary care sites often focus on different metrics depending on current quality improvement initiatives. CHA has developed an ACO-PCMH balanced scorecard to help guide the transition but its leadership uses a different organizational balanced scorecard to track progress with the board.

Cambridge Health Alliancecalls itself a "matrixed" organization-meaning that reporting relationships are both vertical and horizontal to facilitate project teams-but this structure can lead to unclear lines of authority. Three-person teams made up of a medical director, nurse leader, and practice manager lead each primary care clinic. At the time of this case study, a leadership team of the same structure was responsible for systemwide primary care oversight. The members of these teams report up through three departments: medicine, nursing, and operations. Some site leaders have noted conflicting priorities from different parts of the organization-for example, whether they should focus on generating volume or on redesigning the care model. As such, CHA has recently restructured its oversight for primary care with a single senior vice president to ensure accountability and decision-making.

\section{High Levels of Substance Abuse, Mental Health Problems, and Social Determinants of Illness}

CHA's patient population faces a myriad of social ills including poverty, housing instability, and transportation issues that fall largely outside the health care sector's purview. They also have multiple language and literacy needs. As a prominent provider of psychiatric and addiction services, CHA sees a disproportionate share of patients with substance abuse and mental health issues. Caring for these patients presents an ongoing long-term expensive challenge unique to the safety net. The nature of these issues makes it difficult to achieve savings in the near term.

\section{Managing the Conflicting Demands of Multiple Initiatives}

Given its resource limitations, CHA often looks outside the organization for funding to support its transformation initiatives. It has aggressively pursued grants, collaboratives, and government programs including state and private PCMH initiatives, Medicare ACO and care transition programs, and the state's DSTI waiver. Applying for these programs and staying on top of the reporting requirements requires a huge investment in staff resources, which can lead to burnout. This is not unique to $\mathrm{CHA}$ as many primary care practitioners feel overwhelmed in dealing with the current challenges of the profession, but the level of stress is heightened by CHA's resource limitations, the challenges 
facing its patient population, and the imperative to change quickly. Physicians and clinic staff are encouraged by the changes and understand that the goal of transformation is not only to improve patient care but also their work environment.

\section{CONCLUSIONS}

Based on CHA's experience, we have identified several lessons for safety-net providers that are contemplating both accountable care organization and patient-centered medical home transformation.

- Beware of project overload. The vast array of external requirements for safety-net providers offers both opportunities and distractions. Opportunities to get extra funding for transformation inevitably come with an array of reporting and other requirements. Safety-net providers-and, in fact, all providers - and their partners need to consider mechanisms to limit commitments that deplete resources and draw focus away from core transformation efforts.

- Emphasize workforce development. Few safety-net organizations have the resources necessary to build infrastructure and hire new staff while continuing to support their core mission. These limitations increase the importance of workforce transformation to elevate existing staff into more productive roles as part of high-functioning care teams.

- Recruit government partners. The state and federal governments are essential partners if safety-net providers are to engage in a large-scale transformation process. Programs like the Massachusetts DSTI are needed so that safety-net providers can evolve their delivery models to succeed in the new era of accountable care. However, planning for sustainability is essential for future survival.

- Partner with health plans. Delivery systems pursuing transformation can benefit from health plan partners that work with them to provide data, conduct performance analysis, and support new systems that can subsequently be spread across the organization. CHA used its first major global payment contract as a platform for developing its complex care and referral management systems-both are critical to the broader ACO initiative.

- A hospital may be critical for survival. For safety-net ACOs, having a hospital may be critical for economic viability since Medicaid disproportionate share payment formulas are linked to hospital utilization. This reality may alter the way that safety-net providers develop their integrated systems.

- Different approaches are needed for safety-net populations. While the general principles and strategies of the PCMH model are useful in transforming care, these models must be adapted to accommodate the characteristics of safety-net populations in the following ways: empowering frontline staff from the community - particularly medical assistants — to take on expanded roles within the care team assisting physicians, rather than relying on more expensive staff for these functions; establishing complex care management teams that include social workers and community resource specialists who can address social determinants of poor health; and establishing universal screening for depression and protocol-based mechanisms to follow up with patients who have been diagnosed. 


\section{NOTES}

1 C. Luband, ACOs and Medicaid: Challenges and Opportunities (Boston: Ropes \& Gray LLP, 2011).

2 A. G. Raymond, Lessons Learned from the Implementation of Massachusetts Health Reform (Boston: Blue Cross Blue Shield Foundation of Massachusetts, 2011).

3 K. Leighton, E. Jones, P. Shin et al., "Safety-Net Providers after Health Care Reform: Lessons from Massachusetts," Archives of Internal Medicine, Aug. 2011 171(15):1379-84.

4 R. Mechanic, P. Santos, B. E. Landon et al., "Medical Group Responses to Global Payment: Early Lessons from the 'Alternative Quality Contract' in Massachusetts," Health Affairs, Sept. 2011 30(9): 1734-42.

5 A. Gosline and E. Rodman, Summary of Chapter 224 of the Acts of 2012 (Boston: Blue Cross Blue Shield Foundation of Massachusetts, 2012).

6 Ibid.

7 J. Bigby, December 201115 Waiver Approval, 2011.

8 D. M. Berwick, T. W. Nolan, and J. Whittington, "The Triple Aim: Care, Health, and Cost," Health Affairs, May 2008 27(3):759-69.

9 Cambridge Health Alliance, Annual Financial Statement, June 30, 2012.

10 A. Boros, Health Care Provider Price Variation in the Massachusetts Commercial Market (Boston: Center for Healthcare Information and Analysis, Feb. 2013).

11 J. Unutzer, W. Katon, C. M. Callahan et al., "Collaborative Care Management of Late-Life Depression in the Primary Care Setting: A Randomized Controlled Trial," JAMA, Dec. 2002 288(22):2836-45.

12 Cambridge Health Alliance, PCMH Workforce Survey, (Cambridge, Mass.: CHA, 2013).

\section{ABOUT THIS STUDY}

This case study used a mixed-methods approach that included: qualitative interviews; internal document review; assessment of the external market context; and analysis of per-capita spending for CHA enrollees in risk contracts before and after global payment. Thirty semi-structured interviews and five group interviews were conducted with leaders and staff throughout the organization to examine specific CHA initiatives implemented at local practice sites. Transcripts were reviewed for thematic content related to the major research questions.

Researchers had access to corporate scorecards, program applications, financial reports, and ambulatory quality metrics from the prior five years. An interrupted time series of per capita spending before and after global payment was conducted for CHA patients enrolled in a Medicaid managed care plan and compared to spending trends in the rest of the insurer's network.

An advisory group of CHA leaders provided ongoing feedback and validation of results. 


\section{ABOUT THE AUTHORS}

Karen Hacker, M.D., M.P.H., is the director of the Allegheny County Health Department in Pittsburgh, Pa. Dr. Hacker was formerly the executive director of the Institute for Community Health and senior medical director for public and community health at the Cambridge Health Alliance. Her research has focused on community and public health and, more recently, primary care delivery. She has authored reports and peer-reviewed articles on a range of health-related topics and recently published a book on community-based participatory research. She received her medical degree from Northwestern University and her master's in public health from Boston University.

Robert E. Mechanic, M.B.A., is senior fellow at the Heller School of Social Policy and Management at Brandeis University, where his research focuses on health care reform, payment systems, and the adaptation of organizations to new financial incentives. He previously was senior vice president with the Massachusetts Hospital Association and vice president with the Lewin Group. Mr. Mechanic's work has been published in the New England Journal of Medicine, JAMA, Health Affairs, and Business and Health. He earned an M.B.A. in finance from The Wharton School at the University of Pennsylvania.

Palmira Santos, Ph.D., is a senior policy and research analyst at Brandeis University. Dr. Santos is the primary or coinvestigator on a variety of evaluations focusing on delivery reform, quality, and patient safety initiatives. She has worked extensively as a lead evaluator testing outcome measurement, access and utilization of primary care, pilot medical home models, safety and quality improvement, and national chronic disease prevention and management programs. She completed her doctorate at Brandeis University.

\section{ACKNOWLEDGMENTS}

The authors are grateful for the advice and counsel of the Cambridge Health Alliance Advisory group: Soma Stout, M.D., vice president for patient-centered medical home development; David Bor, M.D., chief of medicine; Douglas Thompson, chief administrative officer for the accountable care organization; Allison Bayer, chief operating officer; and Gerald Steinberg, M.D., chief medical officer. In addition, Mr. Gordon Boudrow, chief financial officer, provided assistance with financial information. We would also like to thank all staff members who participated in qualitative interviews. The authors also thank Adriana Bearse, M.S., and Kathleen Xu, M.P.H., of the Institute for Community Health, as well as Umbereen Nehal, M.D., for their work on the case study.

\section{FUNDING SUPPORT}

Support for this research was provided by The Commonwealth Fund. The views presented here are those of the authors and not necessarily those of The Commonwealth Fund or its directors, officers, or staff. 

This study was based on publicly available information and self-reported data provided by the case study institution(s). The Commonwealth Fund is not an accreditor of health care organizations or systems, and the inclusion of an institution in the Fund's case studies series is not an endorsement by the Fund for receipt of health care from the institution.

The aim of Commonwealth Fund-sponsored case studies of this type is to identify institutions that have achieved results indicating high performance in a particular area of interest, have undertaken innovations designed to reach higher performance, or exemplify attributes that can foster high performance. The studies are intended to enable other institutions to draw lessons from the studied institutions' experience that will be helpful in their own efforts to become high performers. It is important to note, however, that even the best-performing organizations may fall short in some areas; doing well in one dimension of quality does not necessarily mean that the same level of quality will be achieved in other dimensions. Similarly, performance may vary from one year to the next. Thus, it is critical to adopt systematic approaches for improving quality and preventing harm to patients and staff. 